\title{
Factores coadyuvantes de la innovación en las cooperativas agroalimentarias. Caso estudio de las citrícolas españolas
}

\author{
R.J. Server-Izquierdo y N. Lajara-Camilleri* \\ CEGEA. Departamento de Economía y Ciencias Sociales, Universitat Politècnica de València. Camino de \\ Vera s/n, 46022 Valencia
}

\begin{abstract}
Resumen
La innovación ha sido reiteradamente señalada como factor de competitividad en la empresa. Como tal, resulta clave estudiar los aspectos que inciden en ella para conducir a la mejora de la posición competitiva en el mercado. Las cooperativas, como entidades con actividad empresarial que son, no escapan de esta realidad. Sin embargo, son escasos los estudios que abordan específicamente el estudio de los recursos internos en las cooperativas agroalimentarias y más aún aquellos que aportan evidencias empíricas. El objetivo de este trabajo es abordar la innovación como fuente de competitividad específicamente en cooperativas agroalimentarias, estudiando los factores que contribuyen a ella. Para ello, tomando como base una muestra de 45 cooperativas citrícolas exportadoras españolas, se estudia la incidencia que la orientación al mercado, la dimensión, la formación y perfil del directivo y la internacionalización tienen sobre la innovación. Los resultados obtenidos muestran una relación de la innovación con el tamaño empresarial, la orientación al mercado y el grado de internacionalización. Se pone de manifiesto una relación apreciable también con el perfil empresarial del gerente aunque el factor de la formación del gerente resulta no significativo, si bien este dato debería matizarse en estudios posteriores para valorar la influencia del conjunto de trabajadores y no sólo del director.
\end{abstract}

Palabras clave: Innovación, competitividad, análisis logit, orientación al mercado, formación.

\begin{abstract}
Factors explaining innovation in agro-food cooperatives: A case study for Spanish citrus cooperatives

Innovation has repeatedly been identified as a factor of companies' competitiveness in the agro-food sector. It is of vital importance to study the aspects that improve competitive positions in the market, and in particular, applied to cooperatives. Few up to date studies have specifically addressed the study of internal resources in agro-food cooperatives and even fewer provide empirical evidence. The aim of this paper is to analyze innovation as a source of competitiveness, in particular, to study the factors that influence agrofood cooperative innovation. To do this, the impact of several factors such as market orientation, size, training and management profile and internationalization on innovation is studied. The data used are from a survey conducted to 45 Spanish citrus exporting cooperatives. Results show that a relationship between innovation and firm size, market orientation and the degree of internationalization exists. In addition, innovation is also related to the business profile of the manager but not related to level of education.
\end{abstract}

Key words: Innovation, competitiviness, logit model, market orientation, formation.

\footnotetext{
* Autor para correspondencia: nalade@cegea.upv.es http://dx.doi.org/10.12706/itea.2016.012
} 


\section{Introducción}

El entorno empresarial actualmente se caracteriza por su elevada exposición al cambio. Las organizaciones precisan para su supervivencia y éxito desarrollar capacidades que les permitan obtener ventajas en el entorno frente al resto de competidores. Se tratan por tanto de factores de competitividad. Las cooperativas se han constituido como actores fundamentales en los primeros eslabones de la cadena de valor de la mayoría de los productos agroalimentarios. En España existen 3.844 entidades agroalimentarias, de las que aproximadamente un $90 \%$ son cooperativas de primer y segundo grado (OSCAE, 2014). En 2012 la facturación del cooperativismo agroalimentario español alcanzó los 25.696 millones de Euros, lo cual supuso un $60 \%$ de la Producción Final Agraria obtenida por el conjunto del sector en España y un $30 \%$ de la Producción Bruta de la Industria Alimentaria Española (OSCAE, 2014).

No obstante, la vertiente social de este tipo de entidades ha provocado en ocasiones que su consideración en estudios de índole económica quede relegada, dando prioridad a las empresas de tipo capitalista. Resulta de interés por tanto estudiar la influencia de distintos factores en las cooperativas con el fin de poder dirigir las políticas hacia el impulso de las iniciativas que inciden más directamente en su competitividad. El objetivo de este trabajo es abordar específicamente la innovación como fuente de competitividad en las cooperativas agroalimentarias, estudiando los factores que contribuyen a ella. Para ello se lleva a cabo una sucinta revisión de los estudios publicados hasta la fecha tanto en empresas mercantiles como en cooperativas.

\section{Material y métodos}

Marco teórico

La innovación es un factor de competitividad en el entorno empresarial (Hernández-Espa-
Ilardo et al., 2011; Tamayo et al., 2015). En cooperativas su relevancia ha sido puesta de manifiesto en los trabajos de Fernández de Bobadilla y Velasco (2008) y Juliá-Igual et al. (2012). Más recientemente Marí-Vidal et al. (2014) señalan la innovación y la formación como una de las oportunidades externas que deben aprovechar las cooperativas para evitar el fracaso empresarial.

Sin embargo, la naturaleza intangible de la innovación dificulta su estudio, ya que suscita diversos modos de medir, valorar y cuantificar el grado de innovación. Esta situación acaba redundando en una diversificación de los tipos de análisis que ralentizan la adopción de decisiones tanto en las propias empresas como a nivel de administración.

El concepto de innovación, tal como recoge el Manual de Oslo (OCDE, 2005), se encuentra en continua evolución. Las sucesivas ediciones que se han realizado de este Manual como propuesta de medición de la innovación han ampliado y actualizado la metodología inicial, siendo un claro reflejo del progreso que experimenta la comprensión del proceso de innovación. La última definición aportada señala que "innovación es la introducción de un nuevo, o significativamente mejorado, producto (bien o servicio), de un proceso, de un nuevo método de comercialización o de un nuevo método organizativo, en las prácticas internas de la empresa, la organización del lugar del trabajo o las relaciones exteriores" (OCDE, 2005).

Se distinguen en consecuencia cuatro tipos de innovación en la empresa; a las ya consideradas clásicas de producto y proceso se añaden la innovación organizativa y la de mercadotecnia. No obstante, esta categorización de tipos de innovación es adaptada por la comunidad científica debido a razones operativas y algunos estudios plantean la medición de la innovación bajo una perspectiva triangular, englobando las innovaciones en comercialización dentro de la caja de organización (Maravelakis et al., 2006 y Benito et al., 2012, en- 
tre otros). Este trabajo adopta este enfoque y establece tres grandes categorías de innovación en la empresa: producto, proceso y organización.

A las anteriormente mencionadas dificultades de conseguir una definición clara y estable del concepto de innovación se une la necesidad de establecer indicadores comunes para cualquier tipología de organización. Las medidas de innovación más habituales suelen utilizar indicadores de input como el gasto en I+D o bien de output ya sea mediante el número de patentes registradas o el número de innovaciones. En empresas de una cierta dimensión estas aproximaciones resultan operativas y los resultados pueden ser una buena aproximación para establecer comparaciones dentro de un mismo sector. El problema surge con las PYMEs, en las que la aplicación de estos indicadores puede conducir a conclusiones erróneas (Maravelakis et al., 2006). En las organizaciones de un tamaño reducido el potencial innovador queda subestimado si se limita al uso de los indicadores tradicionales recogidos en el Manual de Frascati (OCDE, 1980).

A lo largo de los años, la conciencia de esta brecha ha propiciado la propuesta de diversos métodos para la medición de la innovación en PYMEs. Baker y Sinkula (1999) aportan una escala que ha sido ampliamente utilizada pero que con las sucesivas revisiones del concepto de innovación queda incompleta al centrarse únicamente en el producto.

Otra interesante contribución es el PIP-Score que trata de cuantificar la innovación de producto mediante benchmarking (Maravelakis et al., 2006). Se definen 30 items, diez correspondientes a producto, diez a proceso y diez a organización, y mediante la respuesta al cuestionario se determina la posición innovadora de la organización. Con una aproximación más tangible, Sempere y HervásOliver (2011) miden el grado de innovación en PYMEs españolas a través del número de innovaciones desarrolladas en los tres últimos años, entendiendo innovación como modelo de utilidad, patente, proceso nuevo o mejorado o producto nuevo o mejorado.

Aragón y Rubio (2005) analizan la innovación en las empresas desde el punto de vista subjetivo del directivo, que valora las acciones respecto a sus competidores e incluyen también la estimación del esfuerzo realizado en llevar a cabo dichas innovaciones.

Específicamente en el ámbito agroalimentario Avermaete (2002) pone de manifiesto la dificultad de estudiar la innovación en base al número de patentes registradas o gasto en $I+D$, debido a tratarse de un sector con un perfil tecnológico más bajo, al menos hasta la fecha. De hecho, las principales innovaciones han sido introducidas por multinacionales (Fayos et al., 2011). Triguero et al. (2013) también subrayan esta cuestión y proponen utilizar una combinación de indicadores para solventar parcialmente el problema de la medición de innovación en PYMEs. Cabe destacar que esta medición de la innovación se ha llevado a cabo en algunos estudios a través de indicadores ad-hoc como la aplicación de normas ISO 9000 o Buenas Prácticas Agrícolas (Melo et al., 2006), con el consecuente inconveniente de perder comparabilidad.

En relación a los factores que propician e impulsan el desarrollo de la innovación en empresas, Jiménez y Sanz (2004) realizan una clasificación de los factores internos que resultan determinantes de la innovación. Destacan la estrategia, la estructura organizativa, la dirección y el liderazgo, los recursos humanos y otros factores como el capital tecnológico y la orientación al mercado.

\section{Hipótesis de investigación}

Tal como se ha indicado anteriormente, el objetivo de este trabajo es analizar los factores que inciden positivamente en la innovación en cooperativas agroalimentarias, tomando como base una muestra de entidades citrícolas. El propósito es determinar la inci- 
dencia o no de aspectos relacionados con la gestión empresarial como la orientación al mercado, la internacionalización, el nivel de formación del gerente y su actitud empresarial así como el tamaño de la empresa y cuantificar la relación que existe, en su caso, entre cada factor y la innovación. Este objetivo se pretende lograr a través del contraste de las siguientes hipótesis de investigación:

\section{Hipótesis 1: Las entidades orientadas al mercado muestran mayor propensión a ser innovadoras}

Uno de los factores internos de la innovación que ha sido objeto de estudio en los últimos años ha sido la orientación al mercado (Narver y Slater, 1990; Grinstein, 2008, Gómez et al., 2009). De hecho, es considerada como un antecedente del proceso de innovación, ya que las organizaciones orientadas al mercado requieren innovación para ser capaces de mantener sus ventajas competitivas (Cambra et al., 2011). Es más, al monitorizar las necesidades de los clientes se induce el proceso innovador.

Los trabajos realizados han estado normalmente referidos a empresas mercantiles y son escasos los estudios empíricos basados en cooperativas. Cabe recordar que la cooperativa, por su estructura de propiedad, sufre de una importante dualidad en su orientación al considerar al socio también como cliente (Juliá et al., 2009). No obstante, en referencia a cooperativas, Bijman (2010) señala que la orientación al mercado fuerza a operar de forma más estratégica, empujando hacia la innovación. En este sentido, dentro del ámbito agroalimentario español, Cambra et al. (2011) llevan a cabo un ambicioso estudio sobre una muestra de 71 bodegas vitivinívolas -algunas de ellas cooperativas- inscritas en la denominación de origen de Aragón (España). Sus resultados confirman la relación positiva entre la orientación al mercado y la innovación.

Este trabajo trata por tanto de proporcionar evidencia empírica sobre esta relación, especí- ficamente en la figura de la cooperativa agroalimentaria.

Hipótesis 2: La condición de empresa innovadora depende del tamaño de la empresa. Las empresas de mayor dimensión suelen ser más innovadoras

La relación entre la dimensión de las organizaciones y su capacidad de innovación ha sido un tema recurrente en la literatura. Damanpour (1992) demostró a través de un metaanálisis de 20 estudios que la relación entre tamaño empresarial e innovación es estrecha si bien está modulada por aspectos como el tipo de organización. En concreto la correlación era mayor en organizaciones con ánimo de lucro frente a las que no son y también existe un vínculo mayor en empresas manufactureras frente a las de servicios. Camisón-Zornoza et al. (2004) replicaron y ampliaron el meta-análisis de Damanpour obteniendo resultados similares en cuanto a la relación entre dimensión e innovación pero contrarios en cuanto al comportamiento al considerar el sector.

Por ello es necesario matizar que la relación entre la innovación y el tamaño empresarial se ha demostrado especialmente cuando la capacidad de innovación se circunscribe al ámbito tecnológico-industrial (Buesa y Molero, 1998). Tal como se ha apuntado anteriormente, dentro del sector agroalimentario las principales innovadoras han sido protagonizadas por multinacionales, lo que corrobora los hallazgos de Damanpour.

No obstante, diversos autores señalan la ventaja que supone la mayor flexibilidad de las PYMEs, que les permite incorporar más fácilmente cambios en sus operaciones (OCDE, 2000).

En definitiva a la luz de lo expuesto, parece existir todavía cierta controversia sobre la influencia del tamaño empresarial en la capacidad de innovación (Metz et al., 2007), este hecho unido a la carencia de estudios 
empíricos sobre cooperativas agroalimentarias sugiere la incorporación de esta hipótesis a la investigación.

Hipótesis 3: Las empresas con un mayor porcentaje de ventas en mercados exteriores son más innovadoras

La relación entre internacionalización e innovación es estrecha y ha sido puesta de manifiesto en estudios teóricos y empíricos (Cavusgil y Knight, 2014).

El enfoque asumido en este trabajo parte sin embargo de cooperativas que son exportadoras, por lo que en ellas la internacionalización no constituye un objeto de innovación. El propósito es contrastar si, de acuerdo a Katsikeas et al. (2000) y Leonidou (1998), la internacionalización y la innovación son aspectos empresariales relacionados de forma significativa y directa, de forma que una mayor exposición a mercados exteriores motiva comportamientos más innovadores en las cooperativas.

Hipótesis 4: Las empresas con directivos con una formación especializada son más innovadoras

La influencia de la formación de los directivos de las empresas en los resultados obtenidos por las mismas es una cuestión que ha sido abordada y contrastada en diversos sectores y tipologías empresariales, también en el ámbito de la economía social (Del Águila y Padilla, 2010).

En relación con la posición competitiva de las empresas en general, Díaz-Fernández et al. (2014) sostienen que el capital humano (incluyendo no sólo capacidades y habilidades sino también nivel educativo) es un factor clave de competitividad en la empresa. Más recientemente, Marí-Vidal et al. (2013) confirman que la formación es uno de los pilares de la competitividad también en el cooperativismo agroalimentario.
Específicamente en relación a la innovación, Datta y Guthrie (1994) ponen de manifiesto una correlación positiva entre los niveles de formación de los directivos y la propensión de la empresa al cambio. Levenburg et al. (2006) estudiaron la relevancia de diversas características del perfil del directivo como la formación, el estilo de liderazgo o la edad en relación a la implementación de innovaciones de proceso u organizativas (adopción de estrategias de eBusiness) en una muestra de PYMEs estadounidenses; su estudio pone de manifiesto que únicamente la formación tiene incidencia significativa $(a l 0,01)$. Este resultado corrobora las conclusiones de Hausman (2005).

Benito et al. (2012) en un estudio específico sobre la incidencia de los factores internos en la innovación de microempresas sostienen que es la formación específica, y no la formación académica general, la que fomenta una mejor utilización de los recursos, favoreciendo en definitiva la capacidad innovadora de las organizaciones. La relación positiva entre formación e innovación se demuestra también en entidades de economía social es los estudios de Del Águila y Padilla (2010), Morales y García (2007) y Borra et al. (2005), en los que se pone de manifiesto que las empresas más innovadoras cuentan con un mayor porcentaje de personal cualificado.

En consecuencia se pretende analizar si las cooperativas citrícolas cuyos directivos cuentan con formación específica son más innovadoras que aquellas cuyos directivos cuentan con un nivel de estudios inferior.

Hipótesis 5: Los directivos de las empresas innovadoras son más agresivos, en términos empresariales, que los de las empresas no innovadoras

Tal como afirman Del Águila y Padilla (2010), el apoyo de la dirección de la organización es uno de los factores más importantes en los procesos de innovación en las empresas. Chat- 
terjee (2014) señaló el papel fundamental que desempeña el líder del proyecto dentro del proceso de innovación en las empresas. En efecto, Gupta y Govindarajan (1984) apuntan que en concreto la disposición a asumir riesgos (willingness to take risks) de los directivos de las empresas favorece las estrategias de expansión mediante nuevos productos o a través de nuevos mercados. Esta afirmación ha sido contrastada en numerosas ocasiones tanto en el plano de la internacionalización de la empresa como en la innovación desarrollada. El bajo nivel de profesionalización que se observaba en la dirección de las cooperativas agroalimentarias españolas (García y Aranda, 2001) es posiblemente uno de los elementos que ha retrasado el estudio de la influencia de las características personales del directivo en las estrategias empresariales de las cooperativas. A pesar de existir excelentes análisis sobre el nivel de formación en las cooperativas (Mozas y Rodríguez, 2003; MaríVidal et al., 2013, entre otros), no hay evidencias sobre el efecto que la aversión al riesgo o la agresividad empresarial del gerente pueden tener sobre la actividad en cooperativas. La agresividad empresarial se entiende como una cierta capacidad de anticipación por parte del empresario (Del Río y Varela, 2006). Mide por tanto la proactividad del directivo ante el contexto socioeconómico en el que la empresa (cooperativa) opera. En base a lo anterior parece oportuno abordar esta cuestión y su influencia en concreto en la capacidad de innovación de las cooperativas.

\section{Metodología y variables}

La regresión logística o análisis logit es una técnica de regresión que se utiliza cuando la variable dependiente que se pretende predecir y explicar es de tipo binario. Según Hair et al. (1999) la regresión logística cuenta con ciertas ventajas frente al análisis discriminante, principalmente relacionadas con la menor exigencia de los supuestos de normalidad de las variables y de igualdad de matrices de varianzas covarianzas. Esta metodología ha sido ampliamente utilizada en estudios económicos de empresa cuando se trata de obtener una predicción o de modelizar un comportamiento de respuesta dicotómica. Mora (1994) utiliza el análisis logit para establecer modelos de predicción del fracaso empresarial y Carrasco y García (2005) aplican un modelo logit para predecir el comportamiento de índices bursátiles e identificar los atributos financieros relevantes en el valor de la empresa. En el ámbito agroalimentario este análisis ha sido también utilizado por Khanal y Mishra (2014) al estudiar las estrategias de supervivencia en explotaciones agroturísticas; Golovina et al. (2014) lo aplican en el estudio del éxito de cooperativas rusas, Rebelo et al. (2000) llevan un estudio análogo en cooperativas vitivinícolas portuguesas y Huiban y Bouhsina (1998) estudian el rol de la innovación en las empresas agroalimentarias francesas, incluyendo cooperativas, a través de una regresión logística. Más recientemente Meliá-Martí y Martínez-García (2015) aplican el análisis logit en cooperativas agrarias para evaluar el grado de influencia de diversos factores en los procesos de fusión.

Como se ha señalado anteriormente, la regresión logística necesita una variable dependiente de tipo dicotómico. En este estudio, siguiendo la medición realizada por Aragón y Rubio (2005), la variable innovación recoge la clasificación como cooperativa innovadora o no (1/0) que realizan los gerentes o directores comerciales de las cooperativas de la muestra.

Las variables independientes se han establecido a partir de las hipótesis de investigación formuladas (Tabla 1).

La medición de la orientación al mercado se ha realizado utilizando la adaptación de la escala MARKOR para cooperativas realizada 
Tabla 1. Definición de las variables independientes

Table 1. Explanatory variable definitions

\begin{tabular}{|c|c|c|}
\hline Variable & Referencias & Respuesta \\
\hline \multicolumn{3}{|l|}{ Hipótesis 1} \\
\hline Orientación al mercado & Arcas (2002) & $0-100$ \\
\hline \multicolumn{3}{|l|}{ Hipótesis 2} \\
\hline \multirow[t]{4}{*}{ Dimensión empresarial ${ }^{1}$} & Rothwell (1983) & 1 (Micro) \\
\hline & & 2 (Pequeña) \\
\hline & & 3 (Mediana) \\
\hline & & 4 (Grande) \\
\hline \multirow[t]{6}{*}{ Volumen de negocio anual } & & 1 (menos de $1 \mathrm{M} €$ ) \\
\hline & & $2(1-2 M €)$ \\
\hline & & $3(2-10 M €)$ \\
\hline & & $4(10-43 M €)$ \\
\hline & & $5(43-50 M €)$ \\
\hline & & 6 (más de $50 \mathrm{M} €$ ) \\
\hline \multirow[t]{6}{*}{ Balance general anual } & & 1 (menos de $1 \mathrm{M} €$ ), \\
\hline & & $2(1-2 \mathrm{M} €)$ \\
\hline & & $3(2-10 \mathrm{M} €)$ \\
\hline & & $4(10-43 \mathrm{M} €)$ \\
\hline & & $5(43-50 \mathrm{M} €)$ \\
\hline & & 6 (más de 50M€) \\
\hline \multirow[t]{4}{*}{ Trabajadores } & & 1 (menos de 10$)$ \\
\hline & & $2(10-50)$ \\
\hline & & $3(50-250)$ \\
\hline & & 4 (más de 250) \\
\hline \multicolumn{3}{|l|}{ Hipótesis 3} \\
\hline \multirow[t]{2}{*}{ Peso de las exportaciones en la facturación } & Katsikeas et al. (2000) & 0 (Menos del $50 \%)$ \\
\hline & Leonidou (1998) & 1 (Más del 50\%) \\
\hline
\end{tabular}

${ }^{1}$ Definida en base a la Recomendación 2003/361/CE, de 6 de mayo, sobre la definición de microempresas, pequeñas y medianas empresas. 
Tabla 1. Definición de las variables independientes (continuación) Table 1. Explanatory variable definitions (continuation)

\begin{tabular}{lll}
\hline Variable & Referencias & Respuesta \\
\hline Hipótesis 4 & & \\
\hline Formación del gerente/director comercial & $\begin{array}{l}\text { Benito et al. (2012), } \\
\text { Levenburg et al. (2006) }\end{array}$ & $\begin{array}{l}0 \text { (Formación básica) } \\
1 \text { (Formación superior/ } \\
\text { especializada) }\end{array}$ \\
\hline
\end{tabular}

Hipótesis 5

Agresividad empresarial

Dichtl et al. (1990), Likert (1-5)

Del Río y Varela (2006)

Peso de las exportaciones

1 (menos $10 \%)$

(Ventas extranjero/ventas totales)

$2(10-25 \%)$

$3(25-50 \%)$

$4(50-75 \%)$

5 (más del 75\%) ${ }^{1}$ Definida en base a la Recomendación 2003/361/CE, de 6 de mayo, sobre la definición de microempresas,
pequeñas y medianas empresas.

por Arcas (2002). Esta escala consta de tres bloques principales: generación de inteligencia, respuesta de toda la empresa y diseminación de la inteligencia.

La dimensión empresarial es una variable que se construye a partir de la definición de micro, pequeña, mediana y gran empresa (por exclusión) recogida en la Recomendación 2003/361/CE, de 6 de mayo, sobre la defini- ción de microempresas, pequeñas y medianas empresas. La categoría de pertenencia se determina en función del número de empleados, volumen de negocios anual y balance total de cada empresa. En la Tabla 2 se recoge la descripción de las variables utilizadas tanto para esta clasificación como para posteriormente hacer un somero análisis descriptivo de la muestra.

Tabla 2. Estadísticos descriptivos de la muestra en relación a la dimensión e internacionalización Table 2. Descriptive statistics of the sample related to size and internationalization

\begin{tabular}{lccccc}
\hline & N & Mínimo & Máximo & Media & Desviación estándar \\
\hline Volumen de negocio actual & 42 & 1,00 & 6,00 & 3,186 & 1,052 \\
Balance general anual & 35 & 1,00 & 4,00 & 2,971 & 0,822 \\
No de trabajadores & 45 & 1,00 & 4,00 & 2,978 & 0,690 \\
Peso de las exportaciones & 38 & 1,00 & 5,00 & 4,500 & 1,084 \\
\hline
\end{tabular}


El peso de las exportaciones es una variable de tipo dicotómico definida como 1 para cooperativas cuyas exportaciones suponen más de la mitad de la facturación y 0 para la que no alcanzan dicho valor. La formación del gerente o director comercial, en su caso, es también de tipo dicotómico, tomando valor $1 \mathrm{si}$ ha recibido formación superior y/o especializada y 0 si no se cumple esta cuestión. La agresividad empresarial ha sido valorada también a través de la escala desarrollada por Del Río y Varela (2006) y se valora el grado de acuerdo del gerente o directivo respecto a una serie de afirmaciones relacionadas con su actitud en la toma de decisiones.

\section{Selección de la muestra y caracterización}

El ámbito de estudio es España, por ello, a partir del listado de Organizaciones de Productores de Cítricos proporcionado por el Ministerio de Agricultura, Alimentación y Medio Ambiente de España en marzo de 2014, se han identificado las entidades cuya forma jurídica es la cooperativa, tienen su domicilio en España y se encuentran en activo, su número asciende a 63 cooperativas y constituyen la población del estudio.

La recogida de datos se ha realizado en base a un cuestionario auto-administrado, formado por preguntas de respuesta cerrada o semiabierta, agrupadas en diversos bloques (perfil del encuestado, orientación al mercado, innovación e internacionalización). El cuestionario ha sido contestado por el gerente o el director comercial de las cooperativas entre los meses de junio y septiembre de 2014. Debido al reducido número de entidades no se ha utilizado ningún método de muestreo, el cuestionario ha sido enviado por vía postal a todas las cooperativas. Tras realizar cinco contactos (vía telefónica, postal y visita personal), se ha obtenido una tasa de respuesta del $71 \%$. La muestra está formada por 45 cooperativas, se considera una muestra representativa asumiendo un nivel de confianza del $95 \%$ con un porcentaje de error del $8 \%$.
Las 45 cooperativas españolas que componen la muestra se localizan en un $86,7 \%$ en la Comunidad Valenciana, correspondiendo a Murcia, Andalucía y Cataluña el resto de las entidades. Esta distribución plasma la realidad de la población en la que el $86 \%$ de las Organizaciones de Productores de Cítricos reconocidas son valencianas. Se trata en todos los casos de cooperativas de primer grado que trabajan fundamentalmente con cítricos, si bien un $24,4 \%$ declara comercializar también otros productos (fundamentalmente frutas no cítricas, con el objetivo de completar el calendario de comercialización y mejorar el rendimiento de las instalaciones).

La heterogeneidad de la muestra se pone de manifiesto en las variables de dimensión (volumen de negocios anual, balance total de la empresa, número de empleados), con entidades que facturan menos de un millón de euros y otras que declaran ventas anuales entre los 43 y 50 millones de euros (Tabla 2). No obstante, atendiendo exclusivamente al criterio del número de empleados, el $82,2 \%$ de las entidades se clasificarían como empresas medianas y un $17,8 \%$ serían grandes. El marcado carácter exportador del cooperativismo citrícola queda patente a través del peso de las exportaciones en la facturación que se sitúa por término medio en más de un $50 \%$.

\section{Resultados}

El análisis LOGIT planteado intenta explicar el carácter innovador o no de las cooperativas citrícolas a partir de sus atributos. Como resultado se obtiene un modelo que obtiene la probabilidad estimada sobre la calificación de cooperativa innovadora. A partir del establecimiento de un valor crítico $(0,5)$ esta probabilidad se convierte en unos y ceros. El valor crítico se ha establecido en función de los porcentajes de las categorías en la muestra, que pueden ser considerados similares. 
Las variables que resultan relevantes en la innovación de las cooperativas citrícolas considerando un nivel de confianza del $5 \%$ son la dimensión, la orientación al mercado y la in- ternacionalización, adicionalmente el perfil del directivo en términos de agresividad empresarial es significativo al menos al $8,2 \%$ (Tabla 3).

Tabla 3. Resultados del análisis logit aplicado a la variable innovación en cooperativas Table 3. Results of the logit analysis of innovation in cooperatives

\begin{tabular}{|c|c|c|c|c|c|}
\hline & $\mathrm{B}^{\mathrm{a}}$ & e.e. ${ }^{b}$ & Waldc & P-valor & $\operatorname{Exp}(B)^{d}$ \\
\hline Orientación del mercado & 0,209 & 0,073 & 8,147 & 0,004 & 1,232 \\
\hline Peso de las exportaciones & $-3,620$ & 1,598 & 5,131 & 0,024 & 0,027 \\
\hline Dimensión empresarial & 1,960 & 0,833 & 5,541 & 0,019 & 7,097 \\
\hline Nivel de educación del gerente & $-1,180$ & 1,026 & 1,323 & 0,250 & 0,307 \\
\hline Agresividad empresarial & 2,305 & 1,324 & 3,034 & 0,082 & 10,029 \\
\hline Constante & $-17,887$ & 6,516 & 7,536 & 0,006 & 0,000 \\
\hline \multicolumn{6}{|l|}{ Ajustes del modelo } \\
\hline logaritmo de la verosimilitud & & & 31,488 & & \\
\hline $\mathrm{R}^{2}$ Nagelkerke $\mathrm{e}^{\mathrm{H}}$ & & & 0,657 & & \\
\hline Hosmer-Lemeshow $^{f}$ & & & $4,938(0,0667)$ & & \\
\hline
\end{tabular}

a Parámetro estimado; b Error estándar; ' Significación estadística con la prueba de Wald; d Odd ratio; e Evalúa el poder de explicación del modelo; ${ }^{f}$ Evalúa la bondad de ajuste del modelo logit.

El ajuste del modelo estimado es bueno ya que tanto el análisis chi cuadrado de la variación del logaritmo de la verosimilitud como en la prueba de Hosmer-Lemeshow (Tabla $3)$, demuestran que no existe una diferencia estadísticamente significativa entre las clasi- ficaciones observadas y predichas. Adicionalmente, el logaritmo de la verosimilitud presenta un valor bajo, lo que indica un mejor ajuste del modelo (Hair et al., 1999). Finalmente la matriz de clasificación muestra ratios de acierto muy elevados (Tabla 4), pro-

Tabla 4. Matriz de clasificación obtenida con el análisis logit ${ }^{1}$ Table 4. Classification matrix from the logit analysis

\begin{tabular}{llccc}
\hline & \multicolumn{2}{c}{ Pronosticado } \\
\cline { 3 - 3 } Observado & No innovadora & 16 & \\
\cline { 3 - 3 } Innovación & No innovadora & Innovadora & \\
& Innovadora & 3 & 4 & 80,0 \\
Porcentaje global & & & 22 & 88,0 \\
\hline
\end{tabular}

${ }^{1}$ Valor de corte: 0,500 . 
nosticando de forma acertada ambas categorías, por lo que no son indicativos de producir problemas en la predicción de ninguno de los grupos.

\section{Discusión}

Los resultados obtenidos empíricamente a partir de una muestra de 45 cooperativas citrícolas españolas permiten realizar las siguientes afirmaciones en relación a las hipótesis planteadas.

Se confirma la hipótesis 1 que vincula a las cooperativas innovadoras con aquellas con una mayor orientación al mercado. El análisis Logit pone de manifiesto una relación estadísticamente significativa entre ambas cuestiones, lo que corrobora y amplía las conclusiones de los estudios previos de Cambra et al. (2011) en el sector vitivinícola. La dimensión empresarial, hipótesis de investigación 2, también se ha confirmado como un elemento en relación a la consideración de cooperativa innovadora. Este resultado se alinea con las evidencias aportadas en otras formas jurídicas por Damanpour (1992) y Camisón-Zornoza et al. (2004) y adicionalmente constituye un hito inicial en la investigación sobre cooperativas en este aspecto. Se verifica también la hipótesis de investigación 3 que considera la relación entre innovación e internacionalización (tomando como referencia el peso de las ventas en mercados exteriores). El resultado se une en consecuencia a los estudios que han evidenciado esta relación (Katsikeas et al., 2000 y Leonidou, 1998). Las dos hipótesis restantes se deben rechazar al adoptar un nivel de significación del 5\%. La hipótesis de trabajo 4 que relaciona la formación superior o especializada del directivo con la innovación es rechazada con los datos planteados. Esta cuestión, que ha sido reiteradamente señalada por múltiples estudios, tanto a nivel teórico como em- pírico debe ser analizada con detenimiento. Existen diversas cuestiones que pueden explicar el resultado obtenido, en primer lugar es posible que tal como afirman Benito et al. (2012) sea la formación específica la que tiene una incidencia directa sobre la innovación y que por tanto, al haber agrupado en una variable tanto formación superior (universitaria) como específica, este efecto quede anulado. La segunda explicación se encuentra en línea con lo que sostienen autores como Sempere y Hervás (2011), que señalan que es la formación conjunta de los trabajadores, medida como número de licenciados universitarios, la que tiene incidencia sobre el nivel de innovación de una empresa. En consecuencia al contemplar en este caso únicamente la formación del director/gerente sería coherente que los resultados no reflejen una relación directa ya que no se está contemplando el escenario completo en cuestión de formación.

No obstante cabe destacar que la relación de la innovación con el perfil del directivo en términos de agresividad empresarial -hipótesis 5- es significativa al menos al 8,2\%, por lo que resulta evidente que ésta existe y quizá sería interesante plantear un estudio similar bajo una perspectiva diferente -quizá englobando otros aspectos de la personalidad del directivo- para profundizar en esta vertiente. En definitiva, este trabajo aporta evidencias sobre la relevancia positiva que la dimensión, la internacionalización y la orientación al mercado tienen sobre la innovación en las cooperativas agroalimentarias, específicamente en las citrícolas españolas, lo cual supone un avance en el conocimiento existente sobre la gestión y la competitividad de este tipo de entidades.

Los resultados obtenidos incitan también a un estudio más exhaustivo de la influencia del perfil del directivo en los efectos de la gestión y queda señalada la necesidad de analizar la formación no solo del puesto directivo sino del conjunto de trabajadores. Sin embargo 
cabe destacar que existen ciertas limitaciones que hay que considerar a la hora de ponderar las conclusiones. Como se ha señalado, las conclusiones se derivan del estudio de una muestra de cooperativas exclusivamente citrícolas, por lo que se trata de un subsector muy concreto dentro del panorama del cooperativismo agroalimentario, con las consiguientes particularidades. Adicionalmente hay que hacer constar que el reducido número de la muestra, si bien cumple con el tamaño mínimo para el análisis, ha imposibilitado la división para poder estimar y validar el modelo logit en dos submuestras diferentes, por lo que ambos pasos se han tenido que realizar sobre la misma y ello puede introducir un sesgo al alza en la capacidad predictiva del modelo. El último lugar cabe recordar que la medición del carácter innovador de las entidades se ha realizado en base a la propuesta de Aragón y Rubio (2005), en la que es el propio gerente de la cooperativa quien valora distintos aspectos de la innovación en la organización, lo que introduce un sesgo al no existir un único criterio uniforme que se aplique a todos los individuos de la muestra.

\section{Agradecimientos}

Los autores quieren agradecer a los dos revisores anónimos sus comentarios que han servido para mejorar la precisión y calidad de este artículo.

\section{Bibliografía}

Aragón A, Rubio A (2005). Factores asociados con el éxito competitivo de las PYMEs industriales en España. Universia Business Review 8:38-51.

Arcas N (2002). Contribución de las cooperativas agrarias de segundo grado a la orientación al mercado de sus cooperativas asociadas: efectos en el desempeño de la relación. CIRIEC-España, Revista de Economía Pública, Social y Cooperativa 41: 139-161.

Avermaete, T. (2002). Systems of innovation: the case of small food firms in the EU, DRUID PhD Winter Conference, 17-19 January, Aalborg, Dinamarca. Disponible en: www.druid.dk/conferences/winter2002/gallery/avermaete.pdf (16 de diciembre de 2014).

Baker W, Sinkula JW (1999). Learning orientation and innovation: integrating and extending models of organizational performance. Journal of market focused management 4:295-308.

Benito-Hernández S, Platero-Jaime M, RodríguezDuarte A (2012). Factores determinantes de la innovación en las microempresas españolas: la importancia de los factores internos. Universia Business Review 33: 104-121.

Bijman J (2010). Agricultural cooperatives and market orientation: a challenging combination? En Market orientation: transforming food and agribusiness around the customer. (Eds. A Lindgreen, M Hingley, P Custance) Aldershot: Gower Publishing, p. 119-136.

Borra C, García A, Espasandín F (2005). Empresa, comportamiento innovador y Universidad: el caso de la Economía Social en Andalucía. Estudios de Economía Aplicada 23(3): 583-606.

Buesa M, Molero J (1998). Tamaño empresarial e innovación tecnológica en la economía española. Información Comercial Española, 773: 155-173.

Cambra JJ, Fraj E, Melero I, Sesé FJ, Vázquez R (2011). Orientación al mercado y al aprendizaje en el sector vinícola aragonés. Documento de trabajo - Fundación Economía Aragonesa (FUNDEAR ) n 58: 1-76.

Camisón-Zornoza C, Lapiedra-Alcamí R, SegarraCiprés M, Boronat-Navarro M (2004). A metaanalysis of innovation and organizational size. Organization Studies 25(3): 331-361.

Carrasco E, García E (2005). Aplicación logit a la predicción de rendimientos bursátiles. Investigaciones Europeas de Dirección y Economía de la Empresa 11: 99-113. 
Cavusgil ST, Knight G (2014). The born global firm: an entrepreneurial and capabilities perspective on early and rapid internationalization. Journal of International Business Studies 46: 3-16.

Chatterjee D (2014). Leadership in Innovators and Defenders: the roles of cognitive personality styles. Industry and innovation, 21(5): 430-453.Damanpour $F$ (1992). Organizational size and innovation. Organization Studies 13:375-402.

Datta DK, Guthrie JP (1994). Executive succession: organizational antecedents of CEO characteristics. Strategic Management Journal 15: 569-577.

Del Aguila AR, Padilla A (2010). Factores determinantes de la innovación en empresas de economía social. La importancia de la formación de la actitud estratégica. CIRIEC-España, Revista de Economía Pública, Social y Cooperativa 67 (1): 129-155.

Del Río ML, Varela MC (2006). Características de los directivos y rendimiento exportador en las pymes españolas. ESIC Market 125: 223-254.

Díaz-Fernández M, López-Cabrales A, Valle-Cabrera R (2014). A contingent approach to the role of human capital and competencies on firm strategy. Business Research Quaterly 17(3): 205-222.

Dichtl E, Koeglmayr HG, Mueller S (1990). International orientation as a precondition for export success. Journal of International Business Studies 21(1): 23-40.

Fayos T, Calderón H, Mir J (2011). El éxito en la internacionalización de las cooperativas agroalimentarias españolas. Propuesta de un modelo de estudio desde la perspectiva del marketing internacional. CIRIEC-España, Revista de Economía Pública, Social y Cooperativa 72 (1): 43-72.

Fernández de Bobadilla S, Velasco E (2008) Gestionan mejor la innovación las empresas de economía social que las sociedades anónimas: estudio comparativo de empresas innovadoras vascas. CIRIEC-España, Revista de Economía Pública, Social y Cooperativa 63: 5-37.

García E, Aranda M (2001). Problemas existentes en la profesionalización de la gestión en la sociedad cooperativa. Investigaciones Europeas de Dirección y Economía de la Empresa 7(3): 139-154.
Golovina S, Hess S, Nilsson, J, Wolz A (2014). Social capital in Russian agricultural production cooperatives. Post-communist economies 26(4): 522-536.

Gómez JE, Rialp J, Llonch J (2009). Influencia de la Orientación al Mercado en la Función Empresarial; Su Impacto en la Capacidad de Innovación y en los Resultados de la PYME Española. Revista Internacional de la Pequeña y Mediana Empresa 1: 46-47.

Grinstein A (2008). The Effect of Market Orientation and its Components on Innovation Consequences: a Meta Analysis. Journal of the Academy of Marketing Science 36: 166-173.

Gupta AK, Govindarajan V (1984). Business unit strategy, managerial characteristics and business unit effectiveness at strategy implementation. Academy of Management Journal, 27(1): 25-41.

Hair JF, Anderson RE, Tatham RL, Black WC (1999). Análisis multivariante. $5^{a}$ ed. Prentice-Hall.

Hausman A (2005). Innovativeness among small businesses: Theory and propositions for future research. Industrial Marketing Management 34(8): 773-782.

Hernández-Espallardo, M, Sánchez-Pérez, M, Segovia-López, C (2011). Exploitation- and exploration-based innovations: The role of knowledge in inter-firm relationships with distributors. Technovation 31 (5-6): 203-215.

Huiban JP, Bouhsina Z (1998). Innovation and the quality of labour factor: An empirical investigation in the French food industry. Small Business Economics 10(4): 389-400.

Jiménez JD, Sanz VR (2004). Determinantes del éxito de la innovación. Revista de Empresa 7: 24-28.

Juliá JF, Lajara-Camilleri N, Melia E (2009). Factores de competitividad en las cooperativas agrarias valencianas. La orientación al mercado y las alianzas empresariales. La gestión de empresas de economía social, 37-60.

Juliá Igual JF, Melia-Martí E, García-Martínez G (2012). Strategies developed by leading EU agrifood cooperatives in their growth models. Service Business. An International Journal 6(1): 27-46. 
Katsikeas CS, Leonidou LC, Morgan NA (2000). Firmlevel export performance assessment: review, evaluation, and development. Journal of the Academy of Marketing Science 28(4): 493-511.

Khanal AR, Mishra AK (2014). Agritourism and offfarm work: survival strategies for small farms. Agricultural economics 45: 65-76.

Leonidou LC (1998). Organisational determinants of exporting: conceptual, methodological, and empirical insights. Management International Review 38(1): 7-52.

Levenburg N, Magal SR, Kosalge P (2006). An exploratory investigation of organizational factors and e-Business motivations among SMFOEs in the US. Electronic markets 16(1): 70-84.

Maravelakis E, Bilalis N, Antoniadis A, Jones KA, Moustakis V (2006). Measuring and benchmarking the innovativeness of SMEs: a three-dimensional fuzzy logic approach. Production Planning and Control 17(3): 283-292.

Marí-Vidal S; Lajara-Camilleri N; Server RJ (2013). La formación en las sociedades cooperativas agrarias como factor clave de competitividad en un contexto de concentración e internacionalización de los mercados. Interciencia 38(2): 112-120.

Marí-Vidal S; Marín-Sánchez M; Seguí-Mas E; Michael-Zamorano M (2014). Análisis cualitativo de los determinantes en la predicción del fracaso empresarial en cooperativas agroalimentarias. Información Técnica Económica Agraria, Vol 110(3): 300-320.

Meliá-Martí E, Martínez-García A (2015). Factores críticos del éxito de una fusión de cooperativas. Información Técnica Económica Agraria, 111(1): 73-92.

Melo LI, Melo MM, Rodríguez LF (2006). Competitividad del sistema agroalimentario de la ceboIla de bulbo con enfoque de cadena productiva en el Distrito de riego del Alto Chicamocha (Bocayá). Agronomía Colombiana 24(2): 367-377.

Metz I, Terziovski M, Samson D (2007). Development of an Integrated Innovation Capability Model en Building Innovation Capability in Organizations: an International Cross-case perspective. Imperial College Press.
Mora A (1994). Los modelos de predicción del fracaso empresarial: una aplicación empírica del logit. Revista Española de Financiación y Contabilidad 78: 203-233.

Morales AC, García J (2007). Innovación empresarial y economía social: un estudio empírico a nivel andaluz. Strengthening and Building Communities: The Social Economy in a Changing World. $1^{\text {st }}$ International CIRIEC Research Conference on the Social Economy 22-25 de octubre de 2007 en Victoria, BC, Canadá.

Mozas A, Rodríguez J (2003). La formación de los recursos humanos de las cooperativas agrarias y la nueva economía: un estudio empírico. Boletín Económico del ICE, Información Comercial Española 2776: 9-19.

Narver J, Slater S (1990). The Effect of Market Orientation on Business Profitability. Journal of Marketing, 54: 20-35.

OSCAE (2014). Disponible en: http://www.agroalimentarias.coop/ficheros/doc/04281.pdf

OCDE (1980). La medicion de las actividades científicas y técnicas "Manual de Frascati". Método tipo propuesto para las encuestas sobre investigación y desarrollo experimental. Ed. CDTi, Madrid.

OCDE (2000). Small and Medium-Sized Enterprises: Local Strengths, Global Reach. Policy Brief.

OCDE (2005): Oslo Manual: Guidelines for Collecting and Interpreting Innovation Data, $3^{\mathrm{a} e d .}$ OECD Publishing and Eurostat.

Rebelo J, Caldas, JV, Teixeira M (2000). Economic role, governance structure and labour skills of Portuguese wine co-operatives. Proceedings of $4^{\text {th }}$ International Conference on Chain Management in Agribusiness and the Food Industry, 25-26 Mayo, Wageningen University, Holanda, pp. 533-541.

Rothwell R (1983). Innovation and firm size: a case for dynamic complementarity. Journal for General Management 8(3): 56-67.

Sempere F, Hervás-Oliver JL (2011). ¿Qué explica la innovación en PYMEs? Dirección y Organización 43: 5-15. 
Tamayo JA, Romero JE, Gamero J, Martínez-Román JA (2015). Do Innovation and Cooperation influence SMEs' competitiveness? Evidence from the Andalusian metal-mechanic sector. Innovar, Revista de Ciencias Administrativas y Sociales 25(1): 101-115.
Triguero A, Córcoles D, Cuerva MC (2013) Differences in innovation between food and manufacturing firms: an analysis of persistence. Agribusiness 29(3): 273-292.

(Aceptado para publicación el 17 de abril de 2015) 